\title{
Comunicaciones Integradas de Marketing para la implementación de una cultura inclusiva en la educación universitaria
}

Integrated Marketing Communications for the
implementation of an inclusive culture in university
education


\section{Ximena Vélez Calvo}

Docente Investigadora de la Universidad del Azuay

Doctora en Neurociencia Cognitiva y Educación por la Universidad de Valencia Coordinadora del Programa UDA Accesible de la UDA. Ha colaborado en programas de investigación con Universidades Internacionales ( Pennsylvania State University-USA, Universidad de Córdoba-España, Universidad de Valencia-España y Universidad de San Sebastián-Chile) y Universidades Nacionales (Universidad de Cuenca, ESPOL, PUCE, Universidad Central del Ecuador, INSPI)

xvelez@uazuay.edu.ec

ORCID: 0000-0002-4451-9547

\section{José Vera-Reino}

Docente Investigador de la Universidad del Azuay

Economista por la Universidad Católica de Cuenca. Master en Negocios con especialización en Marketing por la Universidad de Internacional del Ecuador. Master en Comercio y Marketing por la Escuela de Negocios ISEAD, Madrid - España. Docente Investigador en áreas de emprendimiento, marketing y Administración de Empresas.

jvera@uazuay.edu.ec

ORCID: 0000-0002-4972-7249

\section{Carolina Cabrera Espinoza}

Universidad del Azuay

Ingeniera en Marketing: Universidad del Azuay. Cuenca- Ecuador. Coordinadora de Proyectos de Investigación con Entrepreneur Colombia. Jefe de Ventas: Grupo Ideas en Cuenca. Gerente - Propietario: Franquicia "Mundo Light" en Cuenca. Jefe de Mercadeo: Lácteos "Los Pinos". Asesoría para la consecución de fondos para empresas privadas. Jefe de Marketing: Networking Marketing y Diseño.

ccabreradar@hotmail.com

ORCID: 0000-0002-9448-4418 


\section{Dayanna Domínguez Bautista}

Universidad del Azuay

Ingeniera en Marketing, Universidad del Azuay, Participación en el programa empresas en acción. Junior Achievement, Cuenca Ecuador, ECASA Cia. Ltda. Auxiliar contable Asesoramiento en marketing y ventas. GPcompu Ingenieria Informatica.

karladominguezbautista@hotmail.com

ORCID: 0000-0003-0348-6141

Fecha de recepción: 19 de julio de 2018 / 3 de septiembre de 2018

\section{Resumen}

Uno de los desafíos de las universidades que tienen declaradas políticas de inclusión, debería ser la puesta en marcha de acciones que apoyen la construcción de una cultura inclusiva. Estas acciones se podrían apoyar en las Comunicaciones Integradas de Marketing. Este estudio buscó determinar las percepciones de la comunidad universitaria respecto a las personas con discapacidad para generar estrategias de CIM que promuevan su inclusión y vinculación. El trabajo se realizó en una universidad privada en Cuenca-Ecuador utilizando metodología mixta. Se observó que la comunidad aún mantiene esquemas mentales que ubican a la discapacidad en el modelo del déficit y percibe los derechos de este colectivo como beneficios. Se propone en consecuencia una campaña de CIM vinculada al modelo de adopción de los aprendizajes la que puede ser una herramienta que facilite el desarrollo de la cultura inclusiva institucional

\section{PALABRAS CLAVE:}

Adopción de los aprendizajes, comunicaciones integradas de márketing, inclusión, percepciones, personas con discapacidad, universidad.

\section{Abstract}

One of the challenges of the universities that have declared policies of inclusion, should be the implementation of actions that support the construction of an inclusive culture. These actions could be supported in the Integrated Marketing Communications. This study sought to determine the perceptions of the university community regarding people with disabilities to generate CIM strategies that promote their inclusion and linkage. The work was done at a private university in Cuenca, Ecuador using mixed methodology. It was noted that the community still maintains mindsets that put disability on the model of deficit and perceived rights of this group as benefits. Therefore, a CIM campaign linked to the model of learning adoption is proposed, which can be a tool that facilitates the development of the institutional inclusive culture.

\section{KEYWORDS}

adoption of learning, integrated marketing communications, inclusion, perceptions, people with disabilities, university. 


\section{INTRODUCCIÓN}

Las Comunicaciones Integradas de Marketing (CIM) pueden ser valiosos recursos que apoyen procesos institucionales que implican desafíos complejos, como es el caso de la inclusión de personas con discapacidad (PD) en las Instituciones de Educación Superior (IES). La inclusión es reconocida como la estrategia clave que permite la educación para todos a través de la presencia, aprendizaje y participación de los miembros de una comunidad educativa (Echeita et al., 2009; Unesco, 2009).

Las CIM se orientan a una integración estratégica del mercadeo para transmitir mensajes claros, consistentes, uniformes y coherentes, integrando los canales de comunicación necesarios que utiliza una institución de forma organizada, coordinada y cuidadosa (Kotler y Armstrong, 2013; Galmés y Victoria, 2012). Estas estrategias son un ventajoso soporte en el desarrollo de las acciones de marketing, pues aportan uniformidad en los mensajes, apoyan la consecución de los objetivos propuestos y garantizan en buena medida la eficacia y el impacto de las acciones propuestas para los grupos objetivo (Santos-Vijande, 2008). Así pues, las estrategias de CIM apoyan en la selección de acciones de mercadeo para que estas puedan influir de mejor manera en la percepción y decisiones de los miembros de una organización (Escobar, 2012).

Estas estrategias son diversas y se clasifican en comunicaciones masivas dirigidas al conjunto del mercado y comunicaciones directas, que son aquellas que parten de una base de datos de los consumidores o adoptantes (Jobber y Fahy 2007). En marketing social las estrategias de CIM pueden ser: 1. La gestión de relaciones, que utilizan las tecnologías de la información con la finalidad de identificar los perfiles de los mejores consumidores para así servirlos y rete- nerlos. 2. La publicidad no pagada, 3. El Marketing directo, que planea y crea acciones de comunicación personalizada para conseguir una respuesta directa del consumidor y establecer un contacto a largo plazo y 4. El Marketing por internet (Azevedo y Pomeranz, 2010; Jobber y Fahy, 2007).

Las CIM tienen una serie de estudios que respaldan su eficacia (Porcu, Del Barrio-García y Kitchen (2012), pues, los usos de estas estrategias mejoran la actitud hacia los productos (Swain, 2004). Aunque no existen estudios previos de la aplicación de las CIM para apoyar el desarrollo de una cultura inclusiva en las IES, las razones antes expuestas demuestran que podrían tener un valioso potencial para la implementación de la cultura inclusiva en los sistemas educativos.

Tengamos en cuenta que existe un escenario sobre el cual las CIM deben operar para la implementación de la cultura inclusiva. Suriá, Bueno y Rosser (2011), destacan que una variable poderosa e influyente que afecta a los estudiantes universitarios con discapacidad son precisamente las barreras mentales de sus miembros. Es que la universidad es considerada una de las instituciones más excluyentes para el ingreso y permanencia de los estudiantes que se encuentran en estas condiciones (Moreno, Rodríguez, Saldaña y Aguilera, 2006).

Si bien estas realidades han generado que las IES planteen políticas para promover la inclusión de las PD, es necesario que no se quede en una declaración de buenas intenciones (Ocampo, 2011). En consecuencia, uno de los desafíos de las universidades, debería ser la propuesta y puesta en marcha de acciones que acompañen y nutran ese cambio institucional, enfocados en el paradigma de la inclusión (Ocampo, 2011). Estas acciones deberán tener como propósito liberar a las PD de esa carga 
histórica de percepciones erradas, que ha frenado su potencial y autonomía y promover su "emancipación y empoderamiento, como sujetos de derechos o ciudadanos sustantivos, transformadores, críticos y propositivos" (Yarza, Rojas y López, 2013, p.43).

El Modelo de Adopción de Aprendizaje del Marketing social, pueden convertirse junto con las CIM en otro aliado de estos cambios institucionales. El proceso de este modelo de Colley, (1961), Kotler y Roberto (1992), plantea tres momentos: "aprender, sentir, hacer". El éxito de este modelo radica en aprender la idea y en función de este proceso previo, desarrollar un comportamiento vinculado con este aprendizaje (Muñoz, 2001). De esta manera, el modelo de Adopción de Aprendizaje puede ser una respuesta valiosa para el cambio de actitudes en la comunidad universitaria y a su vez podría promover la educación inclusiva. Tengamos en cuenta que las actitudes son predisposiciones aprendidas hacia ciertos factores determinados (Auzmendi, 1992), que se originan a raíz de las experiencias de los sujetos (Gal y Garfield, 1997; Gázquez, Pérez, Carrión, Luque y Molero, 2015).

Existen resultados interesantes con respecto a la actitud y percepción de los miembros de la comunidad universitaria en temas de discapacidad de acuerdo a diversos factores. Así pues, los trabajos que han evaluado estas percepciones de acuerdo al sexo, han demostrado que las estudiantes y profesoras suelen tener actitudes más favorables hacia la inclusión (Díaz, 2004; Salinas-Alarcón, y Sánchez-Busqués, 2014), aunque otros trabajos no han encontrado diferencias por el sexo (Cobos y Vélez-Calvo, 2016), o por otro lado, han encontrado en la mujer actitudes más negativas (Suriá, 2012).

Los estudios que han revisado las percepciones y actitudes de acuerdo al tipo de participante, sea este profesor, administrativo o docente, han podido encontrar que los profesores han manifestado cierta "incomodidad" al impartir docencia a los alumnos con discapacidad, situación que se ha mostrado en mayor medida en los de mayor edad (Suriá, 2012), mientras que los alumnos suelen tener disposiciones más positivas (Sánchez, Díaz, Sanhueza y Friz, 2008), especialmente aquellos que han podido convivir con estudiantes en estas condiciones (Suriá et al., 2011).

Otros trabajos que han revisado las representaciones sociales en temas de discapacidad (creencias, valores, suposiciones) de los miembros de la comunidad universitaria (profesores, administrativos y estudiantes), muestran una representación positiva de toda la comunidad universitaria en este tema, aunque ponen en evidencia las limitaciones en la accesibilidad física como una forma de discriminación (Cruz, 2016).

Por otro lado, cuando se ha comparado la percepción de los participantes de acuerdo al tipo de facultad o centro de estudios, no se han encontrado diferencias significativas (Martínez y Bilbao, 2011).

Esta variedad de percepciones en torno a la discapacidad, merece ser revisada para generar respuestas comunicacionales que apoyen y fortalezcan el cambio de una cultura institucional, todo ello en busca de apoyar los procesos de educación inclusiva. Rubio (1999) plantea que esta imagen histórica distorsionada de la discapacidad requiere que, a través de estrategias de comunicación, se generen y difundan percepciones más reales que apoyen el respeto a la diversidad. Del Campo y González (2012) pudieron comprobar que al influir en el ideario participante se pudieron reivindicar las representaciones previas que se tenían sobre las personas con discapacidad intelectual, esto 
a través de campañas de comunicación que se realizaron con estudiantes universitarios. De esta manera, el reto de la construcción del paradigma inclusivo por parte de la universidad, implica que se restructuren tanto las infraestructuras como los procesos simbólicos, interpretativos, culturales y organizativos, por lo tanto, implica también la modificación de las estructuras de pensamiento de los miembros de las comunidades en las que tales cambios se llevan a cabo (Foladori, 2008).

El objetivo del presente trabajo es determinar las percepciones de la comunidad universitaria respecto a las PD para de esta manera, generar estrategias de CIM que promuevan su inclusión y vinculación. Nuestra hipótesis es que existirán percepciones diversas dependiendo del tipo de participante, tal y como lo han demostrado trabajos previos. Sin embargo, nos interesa contrastar los criterios entre profesores, estudiantes, administrativos, tipo de centro entre otros. Estas opiniones servirán de partida para el planteamiento de una propuesta para promover la cultura inclusiva, que vincule las estrategias de CIM con el proceso de adopción de aprendizajes.

\section{MATERIALES Y MÉTODOS}

Como el propósito de nuestro estudio fue determinar las percepciones respecto a las PD, optamos por esta metodología de tipo mixto, pues este tipo de trabajos permiten tener una imagen mucho más completa del fenómeno estudiado (Hernández-Sampieri, Fernández-Collado y Baptista, 2010). El estudio cuantitativo fue de tipo descriptivo, comparativo, transversal y prolectivo mientras que el estudio cualitativo se llevó a cabo por medio de grupos focales.
Este trabajo lo realizamos en la Universidad del Azuay (UDA), una IES de la ciudad de Cuenca-Ecuador, que tiene un financiamiento privado, está compuesta por seis facultades (1. Facultad de Ciencias de la Administración, 2. Facultad de Ciencia Jurídicas, 3. Facultad de Ciencia y Tecnología, 4. Facultad de Diseño, Arquitectura y Arte, 5. Facultad de Filosofía, Letras y Ciencias de la Educación, 6, Facultad de Medicina) y un centro de posgrados. Los miembros de esta comunidad universitaria son 7630 sujetos, entre administrativos, docentes y estudiantes.

\subsection{ESTUDIO CUANTITATIVO}

\subsubsection{PARTICIPANTES}

El estudio se aplicó al total de participantes de la institución ( $\mathrm{N}=7630)$. De este total, respondieron el cuestionario 943 sujetos, de los cuales 54,6\% fueron mujeres. La mayoría de los participantes se encontraron en el rango de edad de 21 a 30 años (65,5\%) y fueron estudiantes (80,7\%). La muestra total comprendió sujetos de las seis diferentes facultades de la universidad y el departamento de Posgrados.

\subsubsection{INSTRUMENTOS}

Utilizamos el cuestionario "Percepciones de la comunidad universitaria respecto a las personas con discapacidad" de Cruz (2016). Este instrumento considera tres categorías respecto a la condición de la discapacidad, las que son: 1. Posibilidades/imposibilidades de los alumnos con discapacidad (i.e. ¿considera que los alumnos con discapacidad están en desventaja académica?) 2. Creencias sobre la normalidad/ anormalidad en la discapacidad (i.e. ¿Cree que los alumnos con discapacidad pueden realizar actividades como los demás compañeros?) y 3. Modelos sobre discapacidad (i.e. ¿la discapa- 
cidad es una tragedia para la persona que la tiene?). Al tratarse de un cuestionario que tendría un uso descriptivo se eliminaron en todas las preguntas la segunda parte que consultaba por qué el participante emitía estos criterios.

El cuestionario original consta de 14 preguntas. Para nuestro estudio eliminamos una de las preguntas porque su planteamiento lo consideramos ambiguo (i.e: ¿La discapacidad está relacionada con un estado mental, físico y a veces transitorio?). Las respuestas se registraron por medio de una escala de Likert de 5 niveles, donde 1 significa muy de acuerdo, y 5 muy en desacuerdo. Las preguntas en el cuestionario original son planteadas en sentido negativo (i.e. La discapacidad presenta un problema para sus familiares) de manera que, a mayor valor (muy en desacuerdo), la persona consultada mostraba una actitud más positiva hacia la pregunta. Sin embargo, tres preguntas estaban planteadas en el cuestionario en sentido inverso, por lo que un menor valor mostraba una mejor actitud. Para los análisis de datos fue necesario invertir estas preguntas para que se mantenga una codificación única para todo el cuestionario.

El cuestionario se sometió a una validación cultural en donde se modificaron las expresiones para adaptarlas al contexto del estudio. El cuestionario final, mostró valores de fiabilidad importantes, con un Alpha de Cronbach de, 82.

\subsubsection{PROCEDIMIENTO}

Se solicitaron los permisos y autorizaciones al Vicerrectorado de Investigaciones de la universidad participante. Con este aval, las encuestas se realizaron a través del programa de Google Formularios, y fueron enviadas desde la plataforma del departamento de Tecnologías de la Información y Comunicación (TICS) de la institución, a los mails de toda la comunidad universi- taria. En la primera parte de esta herramienta se informó del propósito del estudio y se solicitó al participante confirme su deseo de formar parte en esta investigación.

El periodo de recepción de respuestas de las encuestas inició el día martes 5 de diciembre del 2017 a las 16:30 horas, hasta el día viernes 8 de diciembre del mismo año, a las 18:00 horas.

\subsection{ESTUDIO CUALITATIVO}

\subsubsection{PARTICIPANTES}

La distribución y el total de participantes de los grupos focales, están descritos en la Tabla 1.

Tabla 1. Participantes grupo focal

\begin{tabular}{c|c|c|c|}
\multicolumn{2}{c|}{$\begin{array}{c}\text { Con disca- } \\
\text { pacidad }\end{array}$} & $\begin{array}{c}\text { Sin disca- } \\
\text { pacidad }\end{array}$ & Total \\
\hline Estudiantes & 11 & 11 & 22 \\
\hline Personal docente & 11 & 11 & 22 \\
\hline Administrativos & 11 & 11 & 22 \\
\hline Total & 33 & 33 & 66 \\
\hline
\end{tabular}

Estos grupos de estudio estuvieron integrados por participantes pertenecientes a las 6 facultades que tienen la Universidad, más Posgrados.

Se establecieron ciertos criterios de inclusión para los participantes, siendo para el caso del personal administrativo y docentes, contar con un mínimo de un año de antigüedad y tener tiempo completo de labores en la institución. En el caso de los estudiantes debieron estar cursando como mínimo el segundo ciclo del año lectivo 2017 - 2018. Las PD requerían contar con el carnet que certifique esta condición. 


\subsubsection{TEMAS A TRATAR}

Utilizamos la técnica de grupos focales, pues nos permite centrar la atención en un tema específico de estudio y analizar por medio de las interacciones que se dan en este espacio, las opiniones de los participantes (Martínez, 2004). Se definieron los temas a tratar en función de la bibliografía revisada. Estos temas fueron:

- ¿Ha tenido experiencias previas con PD en la universidad? ¿Cómo han sido estas? ¿Cómo es el trato hacia las PD en esta comunidad universitaria?

- ¿Cuáles son las principales barreras que una PD debe superar en esta institución?

- ¿Cuáles son los principales desafíos que esta comunidad universitaria tiene para promover la inclusión de las PD?

\subsubsection{PROCEDIMIENTO}

Todos los asistentes fueron citados previamente y de manera personal para las reuniones que se desarrollaron entre el 21 y el 24 de noviembre del 2017, en dos horarios de 09:00 a 11:00 horas y de 18:00 a 20:00 horas. Se realizó un consentimiento informado previo y se solicitó permiso para grabar su participación en un medio digital, el mismo que posteriormente fue transcrito por los investigadores. Con la transcripción se identificaron unidades de análisis las que permitieron redactar los resultados.

\section{RESULTADOS}

Los resultados de los estudios cuantitativos y cualitativos se presentarán a continuación.

\subsection{ESTUDIO CUANTITATIVO}

Se asignaron 6 categorías de clasificación a los grupos encuestados. Cada uno de los grupos, fueron subdivididos en diferentes subclases (Tabla 2).

Tabla 2. Clasificación de los grupos

\begin{tabular}{|c|c|c|}
\hline Variables & N & $\%$ \\
\hline \multicolumn{3}{|l|}{ Edad } \\
\hline > de 20 años & 142 & 15,1 \\
\hline De 21 a 30 años & 618 & 65,5 \\
\hline De 31 a 40 años & 100 & 10,6 \\
\hline De 41 a 50 años & 44 & 4,7 \\
\hline De 51 a 60 años & 28 & 3,0 \\
\hline$<$ de 60 años & 11 & 1,2 \\
\hline Total & 943 & 100 \\
\hline \multicolumn{3}{|l|}{ Sexo } \\
\hline Hombre & 428 & 45,4 \\
\hline Mujer & 515 & 54,6 \\
\hline Total & 943 & 100 \\
\hline \multicolumn{3}{|l|}{ Tipo de Participante } \\
\hline Estudiante & 761 & 80,7 \\
\hline Administrativo & 76 & 8,1 \\
\hline Docente & 106 & 11,2 \\
\hline Total & 943 & 100,0 \\
\hline \multicolumn{3}{|l|}{ Nivel de instrucción } \\
\hline Primaria & 5 & 0,5 \\
\hline Secundaria & 245 & 26 \\
\hline Tercer nivel & 526 & 55,8 \\
\hline Cuarto nivel & 167 & 17,7 \\
\hline Total & 943 & 100 \\
\hline \multicolumn{3}{|l|}{ Facultad } \\
\hline Ciencia y Tecnología & 150 & 15,9 \\
\hline $\begin{array}{c}\text { Ciencias de la Adminis- } \\
\text { tración }\end{array}$ & 384 & 40,7 \\
\hline Ciencias Jurídicas & 46 & 4,9 \\
\hline $\begin{array}{c}\text { Diseño, Arquitectura y } \\
\text { Arte }\end{array}$ & 94 & 10,0 \\
\hline $\begin{array}{c}\text { Filosofía, Letras y CC de } \\
\text { la Educación }\end{array}$ & 168 & 17,8 \\
\hline Medicina & 32 & 3,4 \\
\hline
\end{tabular}




\begin{tabular}{|c|c|c|}
\hline Posgrado & 69 & 7,3 \\
\hline Total & 943 & 100 \\
\hline $\begin{array}{c}\text { Se auto identifica con } \\
\text { discapacidad }\end{array}$ & 45 & 4,8 \\
\hline Si & 898 & 95,2 \\
\hline No & 943 & 100 \\
\hline Total
\end{tabular}

Luego, se compararon las puntuaciones obtenidas en las preguntas del cuestionario, entre las subclases de cada una de las seis categorías.

Cuando las categorías tuvieron más de dos clases, se aplicó un MANOVA para determinar diferencias entre medias. Cuando existieron sólo dos clases, como en el caso de la variable "Sexo", se aplicó un ANOVA univariado.

\subsubsection{MEDIAS DEL CUESTIONARIO DE LA COMUNIDAD UNIVERSITARIA}

El descriptivo de las medias del cuestionario desarrollado por la comunidad universitaria ( $\mathrm{N}=943$ ) presenta los siguientes resultados ( $\mathrm{Fi}$ gura 1).

Según la estructura del cuestionario, la respuesta 3 implicaba una actitud indiferente hacia la pregunta consultada, lo que al parecer sería la tendencia en este grupo de estudio.

Es necesario aclarar que una respuesta más alta implica una percepción favorable de la condición de discapacidad en este tema. Por ejemplo, si se consulta que las PD pueden realizar tareas como el resto de estudiantes, en medida que esta pregunta se acerque más a 5 que es el máximo valor de la escala Likert, la respuesta de la población es estar en desacuerdo con lo consultado y por lo tanto mostrar una percepción más positiva de la discapacidad en este tema. En conclusión, puntajes más altos muestran una percepción más positiva al tema consultado y puntajes más bajos una percepción más negativa. Es necesario indicar que en las preguntas inversas (i.e. son iguales que sus compañeros) se invirtieron los valores para el análisis de datos con el fin de facilitar la lectura de los resultados.

\subsubsection{EDAD}

EI MANOVA realizado a partir de las puntuaciones obtenidas reveló diferencias significantes entre las seis subclases (Lambda de Wilk $(\lambda)=0,83 ; F=783,03 ; p=<, 05)$. Adicionalmente, la comparación de medias determinó significancia en 7 preguntas. Se reportan los resultados para aquellas preguntas que obtuvieron diferencias significativas al comparar puntajes (Tabla 3).

\subsubsection{SEXO}

Existieron diferencias significativas en las puntuaciones entre hombres y mujeres ( $F=23,587$; $p=<0,05)$. Además, existieron diferencias significativas en las medias de los puntajes obtenidos en cinco preguntas. Como en el caso anterior, se reportan los resultados para aquellas preguntas que obtuvieron diferencias significativas al comparar puntajes (Tabla 4).

\subsubsection{TIPO DE PARTICIPANTE}

En cuanto al tipo de participante, existieron diferencias entre los tres grupos (Lambda de Wilk $(\lambda)=, 048 ; F=1426,22 ; p=<, 05)$. También, existieron diferencias en el puntaje para seis preguntas (Tabla 5). 


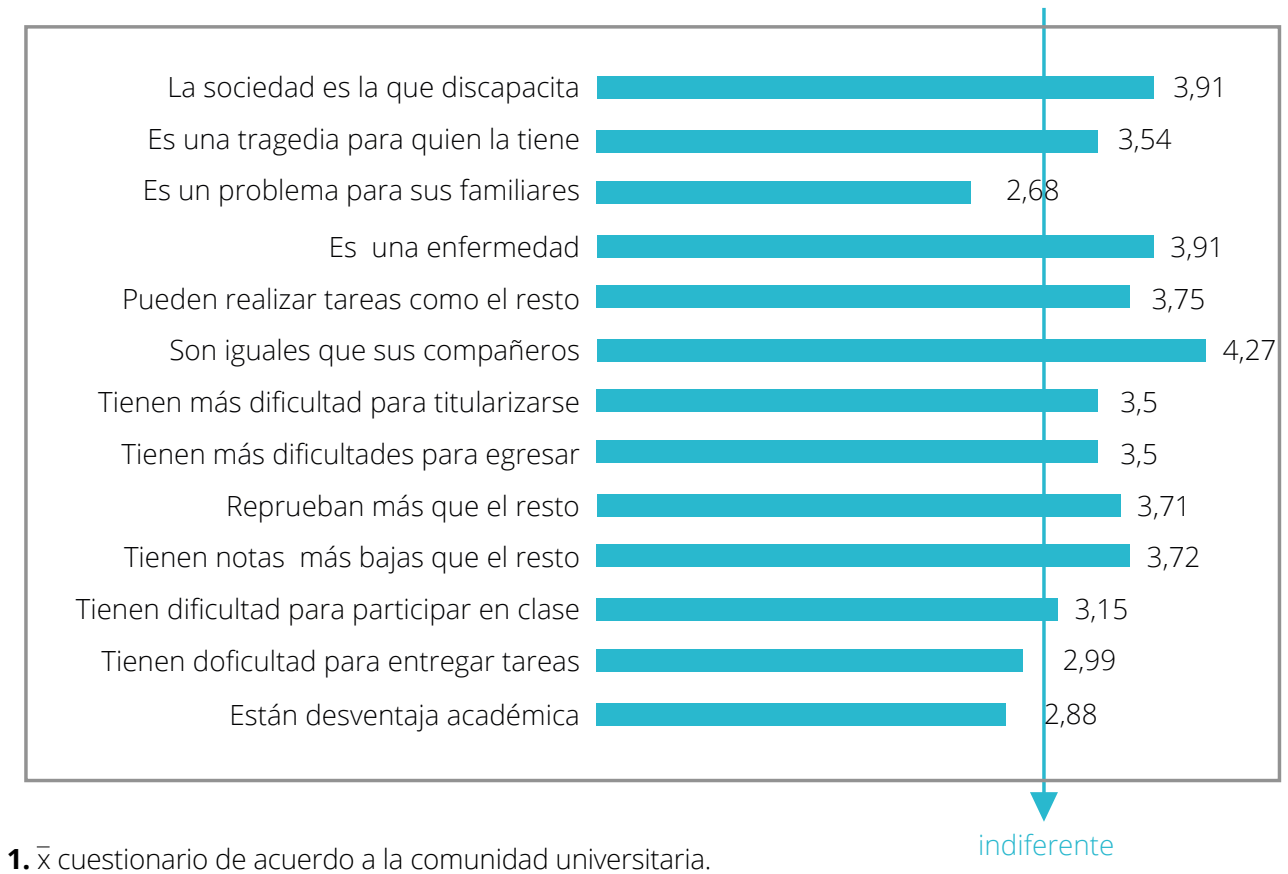

Figura 1. $\bar{x}$ cuestionario de acuerdo a la comunidad universitaria.

Tabla 3. Comparación edad

\begin{tabular}{|c|c|c|c|c|c|c|c|c|c|}
\hline Variables & $\begin{array}{c}<20 \\
\text { años } \\
\bar{x}\end{array}$ & $\begin{array}{c}21 \text { a } \\
31 \\
\text { años } \\
\bar{x}\end{array}$ & $\begin{array}{c}31 \text { a } 40 \\
\text { años } \\
\bar{x}\end{array}$ & $\begin{array}{c}41 \text { a } 50 \\
\text { años } \\
\bar{x}\end{array}$ & $\begin{array}{c}51 \text { a } 60 \\
\text { años } \\
\bar{x}\end{array}$ & $\begin{array}{c}>60 \\
\text { años } \\
\bar{x}\end{array}$ & $F_{(1,942)}$ & $p$ & $\eta_{p}^{2}$ \\
\hline $\begin{array}{l}\text { Tienen dificultad para } \\
\text { participar en clase }\end{array}$ & 2,94 & 3,21 & $3,32++$ & 2,84 & 2,89 & $2,82+$ & 2,787 &, $017 *$ & ,015 \\
\hline $\begin{array}{c}\text { Tienen más dificultades } \\
\text { para egresar }\end{array}$ & 3,37 & 3,56 & $3,61++$ & 3,16 & 3,39 & $2,64+$ & 3,307 &, $006 *$ & ,017 \\
\hline $\begin{array}{l}\text { Tienen más dificultad } \\
\text { para titularizarse }\end{array}$ & 3,40 & 3,56 & $3,57++$ & 3,14 & 3,32 & 2,73 & 3,033 &, $01 *$ & ,016 \\
\hline $\begin{array}{l}\text { Son iguales que sus } \\
\text { compañeros }\end{array}$ & $4,34++$ & 4,32 & 4,1 & 4,16 & $3,54+$ & 4 & 4,769 &, $00 *$ & ,025 \\
\hline $\begin{array}{l}\text { Pueden realizar tareas } \\
\text { como el resto }\end{array}$ & 3,76 & $3,82++$ & 3,53 & 3,61 & $3,25+$ & 3,45 & 2,969 &, 011 * & ,016 \\
\hline Es una enfermedad & 3,60 & 3,93 & $4,09++$ & 4,41 & 3,86 & $3,09+$ & 6,348 &, $00 *$ & ,033 \\
\hline $\begin{array}{c}\text { Es una tragedia para } \\
\text { quien la tiene }\end{array}$ & 3,32 & 3,56 & 3,66 & $3,68++$ & 3,82 & $2,82+$ & 3,633 &, $003 *$ & ,019 \\
\hline
\end{tabular}

$(++)$ media mayor puntuada; (+) media menor puntuada 
Tabla 4. Comparación sexo

\begin{tabular}{|cccccc|}
\hline Variables & Hombre & Mujer & $F_{(1,942)}$ & $p$ & $\eta^{2}$ \\
\hline Pueden realizar tareas como el resto & $\bar{x}$ & $\bar{x}$ & & & \\
\hline Es una enfermedad & 3,67 & 3,82 & 4,51 &, 034 &, 005 \\
\hline Es un problema para sus familiares & 3,71 & 4,07 & 27,83 &, $00 *$ &, 029 \\
\hline Es una tragedia para quien la tiene & 2,6 & 2,75 & 5,58 &, $018^{*}$ &, 006 \\
\hline La sociedad es la que discapacita & 3,37 & 3,68 & 23,92 &, $00 *$ &, 024 \\
\hline
\end{tabular}

Tabla 5. Comparación tipo de participante

\begin{tabular}{|c|c|c|c|c|c|c|}
\hline Variables & $\begin{array}{l}\text { Estudi- } \\
\text { ante } \bar{x}\end{array}$ & $\begin{array}{c}\text { Adminis- } \\
\text { trativo } \\
\bar{x}\end{array}$ & $\begin{array}{c}\text { Docente } \\
\bar{x}\end{array}$ & $F_{(1,942)}$ & $\mathrm{p}$ & $\eta_{p}^{2}$ \\
\hline Están en desventaja académica & 2,92 & $3++$ & $2,53+$ & 4,905 &, $008 *$ & ,01 \\
\hline Tienen dificultad para entregar tareas & 3,02 & $3,09++$ & $2,73+$ & 3,36 &, $035 *$ & ,007 \\
\hline Tienen dificultada para participar en clase & $3,19++$ & 3,16 & $2,88+$ & 3,272 &, $038 *$ &, 007 \\
\hline Tienen más dificultades para egresar & $3,55++$ & 3,47 & $3,16+$ & 6,138 &, $002 *$ & 013 \\
\hline Tienen más dificultad para titularizarse & $3,57++$ & 3,39 & $3,11+$ & 8,744 &, $00 *$ &, 018 \\
\hline Son igual que sus compañeros & $4,32++$ & 4,11 & $4,01+$ & 5,977 &, $003 *$ & 013 \\
\hline
\end{tabular}

$(++)$ media mayor puntuada; $(+)$ media menor puntuada

Tabla 6. Comparación por el nivel de instrucción

\begin{tabular}{|c|c|c|c|c|c|c|c|}
\hline Variables & $\begin{array}{c}\text { Primaria } \\
\bar{x}\end{array}$ & $\begin{array}{c}\text { Bachillerato } \\
\bar{x}\end{array}$ & $\begin{array}{c}\text { Tercer } \\
\text { nivel } \\
\bar{x}\end{array}$ & $\begin{array}{c}\text { Cuarto } \\
\text { nivel } \\
\bar{x}\end{array}$ & $F_{(1,942)}$ & $p$ & $\eta_{p}^{2}$ \\
\hline Es una enfermedad & $2,6+$ & 3,78 & 3,91 & $4,13++$ & 6,09 &, $00 *$ & 0.019 \\
\hline
\end{tabular}

\subsubsection{NIVEL DE INSTRUCCIÓN 3.1.6. FACULTAD}

Existieron diferencias significativas entre los cuatro grupos (Lambda de Wilk $(\lambda)=0,048$; $F=1426,22 ; p=<, 05)$. Adicionalmente, no hubo diferencias en el puntaje en 12 preguntas (Tabla 6).
En cuanto al tipo de facultad, existieron diferencias entre los siete grupos (Lambda de Wilk $(\lambda)=0,034 ; F=2018,008 ; p=<0,05)$. También, existieron diferencias en el puntaje para 8 preguntas (Tabla 7). 
Tabla 7. Comparación tipo de facultad

\begin{tabular}{|c|c|c|c|c|c|c|c|c|c|c|}
\hline Variables & $\begin{array}{l}\text { Cien- } \\
\text { cia y } \\
\text { Tec- } \\
\text { nolo- } \\
\text { gía x- }\end{array}$ & $\begin{array}{l}\text { Cien- } \\
\text { cias } \\
\text { de la } \\
\text { Admi- } \\
\text { nistra- } \\
\text { ción x- }\end{array}$ & $\begin{array}{l}\text { Cien- } \\
\text { cias } \\
\text { Jurídi- } \\
\text { cas x- }\end{array}$ & $\begin{array}{l}\text { Dise- } \\
\text { ño, } \\
\text { Ar- } \\
\text { qui- } \\
\text { tec- } \\
\text { tura y } \\
\text { Arte } x^{-}\end{array}$ & $\begin{array}{c}\text { Filo- } \\
\text { sofía, } \\
\text { Letras } \\
\text { y C. de } \\
\text { la Edu- } \\
\text { cación } \\
\bar{x}\end{array}$ & $\begin{array}{c}\text { Me- } \\
\text { dicina } \\
x^{-}\end{array}$ & $\begin{array}{l}\text { Pos- } \\
\text { grado } \\
x^{-}\end{array}$ & $F_{(1,942)}$ & $p$ & $\eta_{p}^{2}$ \\
\hline $\begin{array}{l}\text { Están en } \\
\text { desventaja } \\
\text { académica }\end{array}$ & 2,77 & 2,95 & 2,61 & 3,03 & $2,55+$ & 3,28 & $3,35++$ & 5,24 &, $000 *$ & ,033 \\
\hline $\begin{array}{l}\text { Tienen dificul- } \\
\text { tad para entre- } \\
\text { gar tareas }\end{array}$ & 2,95 & 3,05 & 2,65 & $2,82+$ & 2,87 & $3,63++$ & 3,22 & 3,67 &, 001 * & ,023 \\
\hline $\begin{array}{l}\text { Tienen dificul- } \\
\text { tad para parti- } \\
\text { cipar en clase }\end{array}$ & 3,11 & 3,12 & $2,89+$ & 3,30 & 2,99 & $3,66++$ & 3,52 & 3,44 &, $002 *$ & ,022 \\
\hline $\begin{array}{l}\text { Tienen notas } \\
\text { más bajas que } \\
\quad \text { el resto }\end{array}$ & 3,67 & 3,72 & 3,65 & 3,86 & $3,50+$ & $4,06++$ & 4,03 & 3,67 &, 001 * & ,023 \\
\hline $\begin{array}{l}\text { Reprueban } \\
\text { más que el } \\
\quad \text { resto }\end{array}$ & 3,72 & 3,71 & 3,80 & 3,73 & $3,52+$ & 3,97 & $4,03++$ & 2,83 &, 010 * & ,018 \\
\hline $\begin{array}{l}\text { Tienen más di- } \\
\text { ficultades para } \\
\text { egresar }\end{array}$ & 3,47 & 3,46 & 3,46 & 3,59 & $3,37+$ & 3,69 & $3,91++$ & 2,49 &, 021 * & ,016 \\
\hline $\begin{array}{l}\text { Tienen más } \\
\text { dificultad para } \\
\text { titularizarse }\end{array}$ & 3,47 & 3,52 & 3,39 & 3,57 & $3,30+$ & 3,66 & $3,87++$ & 2,67 &, $014 *$ & ,017 \\
\hline $\begin{array}{c}\text { Es una enfer- } \\
\text { medad }\end{array}$ & 3,85 & 3,80 & 4,04 & 3,88 & $4,21++$ & $3,44+$ & 4,06 & 4,35 &, $000 *$ & ,027 \\
\hline
\end{tabular}

Tabla 8. Comparación auto identificación por discapacidad

\begin{tabular}{|c|c|c|c|c|c|}
\hline \multicolumn{2}{c}{ Variables } & Si $\bar{x}$ & No $\bar{x}$ & $F_{(1,942)}$ & $\eta^{2}$ \\
\hline Es una enfermedad & 3,60 & 3,92 & 3,88 &, 049 & 4,47 \\
\hline Es un problema para sus familiares & 2,36 & 2,69 & 5,41 &, 020 & 4,93 \\
\hline
\end{tabular}

\subsubsection{SE AUTO IDENTIFICA CON DISCAPACIDAD}

Existieron diferencias significativas en dos preguntas en las puntuaciones entre personas que se identifican con discapacidad y personas que no se identifican con discapacidad ( $F=3,553$; $\mathrm{p}=<, 05)$. (Tabla 8)

\subsection{ESTUDIO CUALITATIVO}

Presentamos los argumentos más recurrentes en función del tipo de participantes, sean estos estudiantes, administrativos y docentes. Para 
el planteamiento de estos resultados se grabaron las respuestas de los grupos focales, se analizaron las grabaciones y se establecieron unidades de análisis.

\subsubsection{ESTUDIANTES}

Los estudiantes opinaron que:

- Los docentes se refieren de forma inapropiada de las PD.

- Es necesario capacitar a los docentes y administrativos para que conozcan las verdaderas razones que limitan funcionalmente a una persona con discapacidad para que se pueda generar las adecuaciones que necesitan para su educación universitaria.

- Las barreras psicológicas y actitudinales, son las que más discapacitan.

\subsubsection{DOCENTES}

El argumento más destacable del grupo focal por parte de los docentes fue que desconocen cómo tratar a un estudiante con discapacidad.

\subsubsection{ADMINISTRATIVOS Y DOCENTES}

Por su parte administrativos y docentes coincidieron en que:

- Las PD abusan de sus derechos.

- Las PD dependen mucho de sus familiares.

\subsubsection{TODOS LOS PARTICIPANTES}

Los argumentos en los que coincidieron todos los participantes fueron:

- La discriminación se ha reducido de forma significativa.
- No hay conocimiento ni difusión de las políticas institucionales de inclusión.

- Crear un ambiente inclusivo también implica la consideración de las necesidades derivadas de la discapacidad, la generación de respuestas y el seguimiento de sus resultados.

- Es necesario difundir los valores y fortalezas de las PD.

\section{CONCLUSIONES Y PROPUESTA}

Este estudio tenía por objetivo conocer las percepciones de la comunidad universitaria respecto a las personas con discapacidad para en función de estas percepciones generar estrategias de CIM que promuevan su inclusión y vinculación

En cuanto a la primera parte de esta investigación que busca establecer las percepciones de la comunidad universitaria, debemos partir indicando que la media general denota una tendencia ligeramente favorable hacia las PD, aunque poco empoderada. Por otro lado, es destacable notar diferencias estadísticamente significativas en ciertas preguntas del cuestionario de Cruz (2016), en donde se aprecia que quienes tienen percepciones más negativas sobre este colectivo son: las personas mayores de 51 años, los de sexo masculino, los profesores, y los participantes de las facultades de Filosofía, Diseño, Ciencias Jurídicas y Medicina.

Trabajos previos realizados en universidades coinciden con algunos de nuestros hallazgos, encontrando mejor percepción y actitudes en el sexo femenino (Díaz, 2004; Salinas-Alarcón, y Sánchez-Busqués, 2014), o en los alumnos (Sánchez et al., 2008). Otros estudios han demostrado que existe cierta resistencia frente a los alumnos con discapacidad en los profeso- 
res universitarios, debido a la falta de motivación o interés que pueden presentar los alumnos con esta condición, así como por la falta de preparación específica del docente (Suria, 2012). Precisamente en nuestro caso, un factor que se manifestó con mucha fuerza en el grupo focal fue que los docentes desconocían como tratar al estudiantado con discapacidad, situación que según el trabajo de Bilbao (2008), ha demostrado ser uno de los factores que limita significativamente el éxito en los logros educativos de estos alumnos.

Además, tanto administrativos como docentes manifestaron en el grupo focal, que los estudiantes con discapacidad abusaban de sus derechos o que dependían de su familia, en lugar de resolver sus propias necesidades como adultos. Esta situación se debería entender desde la perspectiva de las PD, quienes, al tener que enfrentarse a un medio que no puede proveer todos los recursos para apoyarlos y que muestra diversas limitaciones en la accesibilidad, tienen que recurrir al apoyo que pueden encontrar en sus familiares. Probablemente si las instituciones tuvieran mejores condiciones de accesibilidad, tales situaciones no se percibirían de esta manera. Por otro lado, si bien es necesario que las PD se empoderen de su condición, es decir afronten las diversas situaciones de vida, superen las barreras y sean gestoras de sus necesidades y derechos (Suriá y Villegas, 2016; Yarza et al., 2013) estos valores pueden verse limitados por una serie de variables. En efecto, se ha observado que el empoderamiento de estudiantes con discapacidad es menor al compararlo con sus compañeros que no poseen esta condición, por el autoconcepto y la autoimagen que tienen los estudiantes en relación a su discapacidad (Suriá y Villegas, 2016). Sin embargo, es probable factores culturales también estén dificultando el empoderamiento. En este caso, la propia idea que el entorno tiene sobre la discapacidad, puede ser un importante limitador de la independencia, la capacidad de afrontamiento y superación de las PD.

En cuanto a la edad, muchos trabajos han encontrado que los docentes más jóvenes presentan mejores actitudes y por lo tanto mejor percepción en cuanto al alumnado con discapacidad (León y Avargues, 2007). En nuestro caso la comunidad universitaria en el rango de 31 a 40 años, fueron quienes mostraron mejor percepción. Estos hallazgos pueden explicarse por las expectativas positivas de quienes se pueden encontrar en esta etapa etaria, las que probablemente son influenciadas por el modelo de inclusión que el país ha adoptado y que ha sido fuertemente difundido a través de normativas, acciones concretas y políticas puntuales. Al contrario, el grupo de mayor edad fue el que tuvo peor percepción, pues al parecer aún tienen una forma muy conservadora de percibir la discapacidad, situación que coincide con los estudios de Jordan, Lindsay y Stanovich, (1997) quienes concluyen que esta apreciación corresponde a una concepción del modelo del déficit, por lo tanto, perciben a este grupo como desfavorecido, débil y necesitado.

Respecto al tipo de facultad, el centro que peor percepción demostró fue la Facultad de Filosofía a diferencia de otros trabajos que no han encontrado diferencias de acuerdo al tipo de facultad (Martínez y Bilbao, 2013). Las percepciones de los participantes de esta dependencia refieren que: las personas con discapacidad tienen notas más bajas, reprueban más, tiene más dificultades para egresar y para titularizarse, en conclusión, están en desventaja académica. Con la intensión de profundizar más sobre este tema, solicitamos se nos indicara la distribución de estudiantes con discapacidad en las diferentes facultades de esta universidad, para el semestre octubre 2017-marzo 
2018, que corresponde al que se realizó el estudio. Obtuvimos que el $4 \%$ de los estudiantes con discapacidad están en la Facultad de Medicina, $7 \%$ en Ciencias Jurídicas, $11 \%$ en Diseño Arquitectura y Artes, 16\% en Administración de Empresas, 20\% en Ciencia y Tecnología y 42\% en la Facultad de Filosofía. Este alto porcentaje de estudiantes con discapacidad en la Facultad de Filosofía, podría estar explicando las percepciones que este centro de estudios muestra, pues están basadas en las evidencias reales. Entonces, sería valioso revisar las razones de esta desventaja académica, para generar apoyos y soluciones. Esta afirmación se puede complementar con lo mencionado por los participantes del grupo focal, quienes expresaron que se requiere considerar las necesidades que derivan de la discapacidad para que se propongan respuestas y se dé seguimiento a los resultados de las propuestas generadas.

Vale la pena destacar que, la facultad de medicina percibe que la discapacidad es una enfermedad, decantándose con esta apreciación hacia el modelo del déficit. Esta postura se repite, cuando el grupo que no se autoidentifica con discapacidad indica que esta es una enfermedad y que es un problema para sus familiares. Estos resultados demostrarían que el modelo del déficit es la forma de percepción predominante en la comunidad universitaria, por lo tanto las PD son valoradas como incapacitadas y deficientes (Kipen y Lipschitz, 2009)

En respuesta a tales apreciaciones, en el grupo focal los participantes piden que la comunidad universitaria conozca los valores y potencial de las personas con discapacidad y que se difundan las políticas institucionales de inclusión. Estas acciones sugeridas por los participantes, pueden afectar positivamente la presencia, aprendizaje y participación de las personas con discapacidad y probablemente incidir en las barreras mentales que los miembros de la co- munidad universitaria han expresado en este trabajo.

La segunda parte de nuestro objetivo es generar estrategias de CIM que promuevan la vinculación e inclusión de las PD. Para ello vinculamos las estrategias de CIM con el proceso de de Adopción de Aprendizajes de Colley, (1961); Kotler y Roberto (1992).

Las estrategias de CIM buscan a través de los mensajes uniformes y la integración de los canales de comunicación, transmitir mensajes claros, coherentes y uniformes que influyan en los miembros de una organización (Escobar, 2012; Galmés y Victoria, 2012; Kotler y Armstrong, 2013; Santos-Vijande, 2008). Complementariamente, los modelos de adopción de los aprendizajes buscan que el grupo meta aprenda una idea y desarrolle un comportamiento vinculado, en un proceso denominado "aprender, sentir, hacer" (Colley, 1961; Kotler y Roberto, 1992).

Planteamos entonces las siguientes estrategias de CIM de marketing masivo: gestión de relaciones (GR), publicidad no pagada (PNP), marketing directo (MD) y marketing por internet (MI). El proceso que diseñamos está representado en la Figura 2, en donde se demuestra la relación de interdependencia entre el modelo de adopción de los aprendizajes y las CIM.

El objetivo de la campaña resultante es promover actitudes positivas y de apertura de la comunidad universitaria hacia las PD. La campaña se justifica mediante el desarrollo de tres etapas con diferentes actividades interrelacionadas utilizando las CIM antes mencionadas. Las etapas se desarrollarían de la siguiente manera:

Etapa 1 Difundir fundamentos para promover la inclusión de las PD y valorar su potencial. 


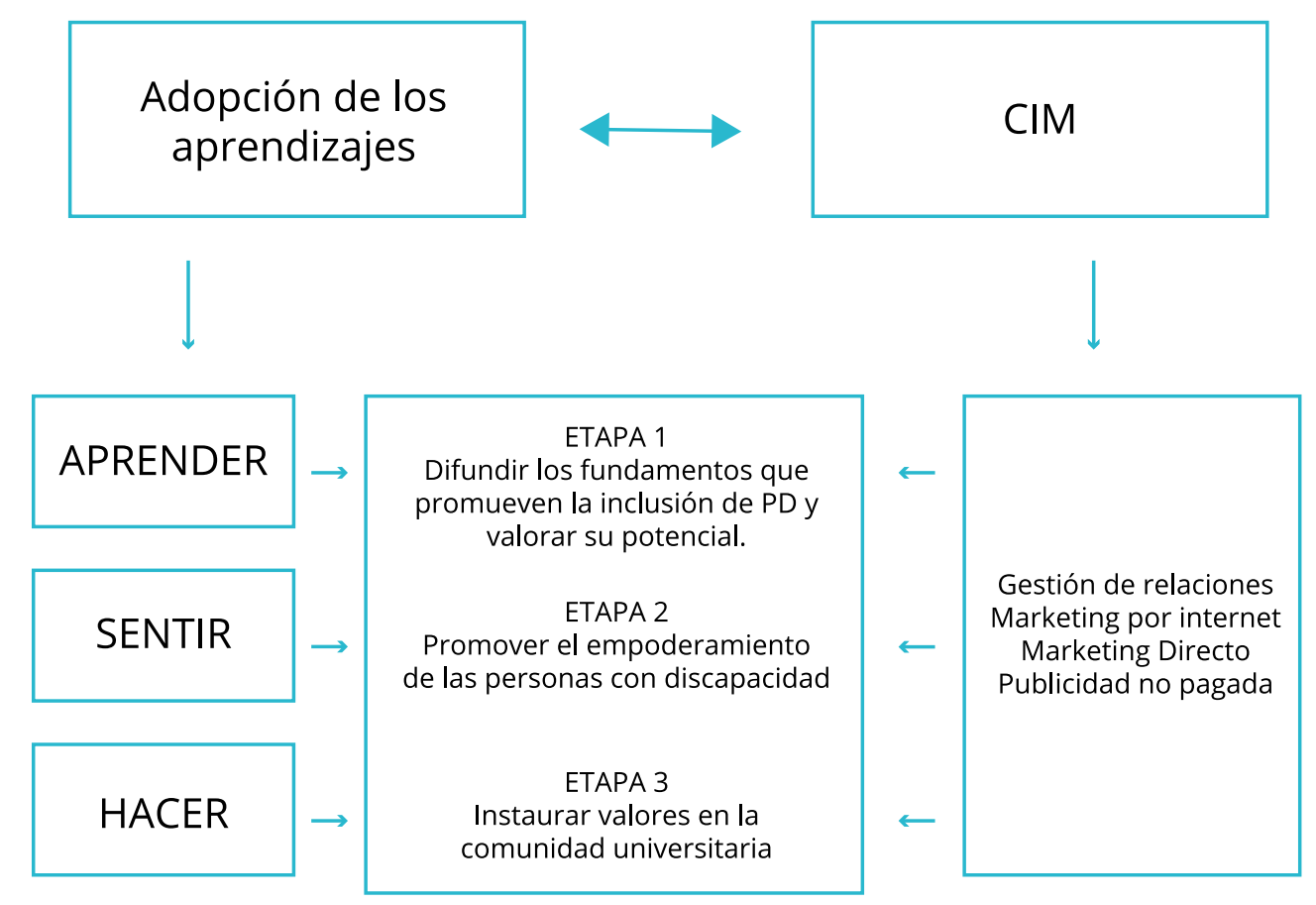

Figura 2. Estrategias de CIM y adopción de los aprendizajes para campaña de vinculación de PD

- Aprender. GR: difundir políticas institucionales de inclusión. Capacitación al personal Docente y Administrativo sobre el tema. MI Y PNP: transmitir íntegramente la campaña en Página Web, redes sociales y radio institucional.

- Sentir. GR: realizar talleres de sensibilización a la comunidad universitaria. MD: activaciones Bellow the line- Ilusión, que permitan entender las barreras que enfrentan las PD. MI Y PNP: trasmitir imágenes de la campaña en página WEB, redes sociales y radio institucional, con el objetivo de sensibilizar y generar reflexiones.

- Hacer: MI Y PNP: Recolectar las opiniones positivas o negativas de todos los involucrados con el "hashtag" de la campaña y difusión en Página Web, redes sociales y radio institucional. GR: Encuestas de validación a los participantes en el semestre posterior a la campaña.
ETAPA 2 Promover el empoderamiento de las PD.

- Aprender. MD: informar cómo se está fortaleciendo la cultura inclusiva en la institución a través del testimonio de las PD. GR: capacitar a la comunidad universitaria sobre el uso de expresiones adecuadas y lenguaje positivo. charlas sobre los derechos de las PD en la comunidad universitaria. MI: feria sobre $\mathrm{TIC}^{\prime} \mathrm{S}$ inclusivas que mejoran el desempeño educativo de las PD.

- Sentir. GR: charla motivacional y experiencial a cargo de PD. atención medica integral para PD. MD: creación del grupo de apoyo "HayUDA" formado por personas de distintas facultades. MPI Y PNP: comunicar el desarrollo de toda la etapa 2 en Página Web, redes sociales y radio institucional

- Hacer: MI Y PNP: Recolectar las opiniones positivas o negativas de todos los involucrados con el "hashtag" de la campaña y difusión en 
Página Web, redes sociales y radio institucional. GR: Encuestas de validación a los participantes en el semestre posterior a la campaña.

ETAPA 3 Instaurar valores en la comunidad universitaria

- Aprender. GR: informar sobre el valor de la actitud positiva hacia las PD.

- Sentir. GR: Generar actividades de impacto y cambio que cultiven inquietudes. MD: Técnica BTL- Intrusión, utilizando objetos, imágenes o frases, en lugares de alto tráfico peatonal con el objetivo de analizar valores y antivalores para la comunidad de PD. MI Y PNP: Comunicar los elementos generados en la técnica de intrusión en Página Web, redes sociales y radio institucional Página Web, redes sociales y radio institucional.

- Hacer: MI Y PNP: Recolectar las opiniones positivas o negativas de todos los involucrados con el "hashtag" de la campaña y difusión en Página Web, redes sociales y radio institucional. GR: Encuestas de validación a los participantes en el semestre posterior a la campaña

De esta manera, el Proceso de Adopción de los aprendizajes nos orienta en la secuencia a seguir, y de este se generan los objetivos para la campaña que se desarrollarán en tres etapas. Por su parte las CIM aportan las estrategias a utilizar, las que hemos desarrollado como acciones.

Esta propuesta busca aprender la idea y desarrollar el comportamiento vinculado (Muñoz, 2001) para afectar las actitudes y percepciones erradas que han existido sobre la condición de discapacidad. Se trata de un modelo que vincula el marketing y la comunicación y que pensamos responderá al reto de mejorar la imagen distorsionada que ha tenido la discapacidad (Rubio, 1999) y que apostamos influirá en las ideas previas de los participantes para favorecer la cultura inclusiva que esta IES persigue y que está declarada en sus políticas institucionales.

\subsection{LIMITACIONES Y RETOS FUTUROS}

Fueron limitaciones de este estudio las siguientes. Por un lado, el cuestionario de Cruz (2016) no presentaba en su versión original una validez declarada, no obstante, los resultados de nuestro trabajo mostraron índices de fiabilidad que demuestran su fortaleza. Otra debilidad es que la mayoría de los participantes del estudio fueron estudiantes $(80,7 \%)$ por lo que las percepciones de docentes y administrativos no podrían considerarse como representativas.

Este trabajo genera una respuesta para afectar las percepciones de la comunidad universitaria respecto a las PD, el que valdría la pena ser validado y aplicado en centros universitarios para determinar si puede afectar las percepciones del colectivo universitario, así como determinar el impacto de las acciones que proponemos. Este trabajo también podría replicarse en otras instituciones y así comparar como factores culturales afectan los resultados. 


\section{BIBLIOGRAFÍA}

Auzmendi, E. (1992). Las actitudes hacia la matemática Estadística en las enseñanzas medias y universitarias. Bilbao: Mensajero.

Azevedo, A., y Pomeranz, R. (2010). Obsesión por el cliente: cómo obtener y retener clientes en la nueva era del marketing relacional. México D.F: McGraw-Hill Interamericana.

Bilbao, M. C. (2008). La integración de personas con discapacidad en la educación superior. Percepciones y demandas de docentes y estudiantes en la Universidad de Burgos. Área Abierta, (31), 1-18.

Cobos, D., Vélez-Calvo, X. (2016). Actitudes de los docentes universitarios hacia las personas con discapacidad. Caso de una universidad privada de Cuenca-Ecuador. Revista del Congreso de Ciencia y Tecnología, 364-367.

Colley, R. (1961). Defining Advertising Goals for Measured Advertising Results. New York: Association of National Advertisers.

Cruz, R. (2016). Percepciones sobre la inclusión de alumnos con discapacidad en la Universidad Veracruzana. REencuentro, Análisis de Problemas Universitarios, 72, 151-178.

Del Campo, A., y González, R. (2012). Comunicación inclusiva: Una experiencia en creación de campañas sobre discapacidad intelectual. Área Abierta, 12(1), 1-18.

Díaz, G. (2004). Los estudiantes con discapacidad en la Universidad de las Palmas de Gran Canaria. Las Palmas de Gran Canarias: Servicio de Publicaciones de la Universidad.

Echeita, G., Simón, C., Verdugo, M., Sandoval, M., López, M., Calvo, I., y Gonzales-Gil, F. (2009). Paradojas y dilemas en el proceso de inclusión en España. Revista de Educación, 349, 153-178.

Escobar, N. (2012). Las comunicaciones integradas de marketing (CIM) como pilar de la estrategia de marketing verde y sus implicaciones en la gestión ambiental. Revista Facultad de Ciencias Económicas: Investigación y Reflexión, (2), 69-79. 
Foladori, H. (2008). La intervención institucional. Hacia una clínica de las instituciones. Santiago: Ediciones ARCIS.

Gal, I., y Garfield, J. (1997). The assessment challenge in statistics education. Amsterdam: IOS press.

Galmés, M., y Victoria, J. (2012). La organización de eventos en el contexto de las Comunicaciones Integradas de Marketing (IMC): el valor de la experiencia. Pensar la publicidad, 6(1), 15-34.

Gázquez, J; Pérez, M; Carrión, J; Luque de la Rosa, A., y Molero, M. (2015). Perfiles de valores interpersonales y análisis de conductas y actitudes sociales de adolescentes. Revista de Psicodidáctica. 20(2), 321-337.

Hernández-Sampieri, R., Fernández-Collado, C., y Baptista, L. (2010). Metodología de la Investigación. México: McGraw Hill.

Jobber, D., y Fahy, J. (2007). Fundamentos de marketing (2a. ed.). Madrid: McGraw-Hill.

Jordan, A., Lindsay, L. y Stanovich, P. J.(1997). Classroom teacherś instructional interactions with students who are exceptional, at risk and typically achieving. Remedial y Special Education, 18, 82-93.

Kipen, E., y Lipschitz, A. (2009). Demasiado cuerpo. En A. Rosato y M. Angelino (Coords.), Discapacidad e ideología de la normalidad. Desnaturalizar el déficit (pp. 117-13). Buenos Aires, México: Noveduc.

Kotler, P., y Armstrong, G. (2013). Principles of Marketing (15a. ed.). New York: Pearson.

Kotler, P. y Roberto, E. (1992). Marketing Social. Estrategias para cambiar la conducta pública. Madrid: Ediciones Díaz de Santos.

León, J.M. y Avargues, M.L. (2007). Evaluación del estrés sociolaboral en el personal la Universidad de Sevilla. Revista Mapfre Medicina, 18, 323-332

Low, G. (2000). Correlates of integrated marketing communications. Journal of Advertising Research, 40 (3), 27-39.

Martínez, M., y Bilbao, M. (2013). Los docentes de la universidad de Burgos y su actitud hacia las personas con discapacidad. Revista Española sobre discapacidad, 42(4), 50-78.

Moreno J., Rodríguez I., Saldaña, D., y Aguilera, A. (2006). Actitudes ante la discapacidad en el alumnado universitario matriculado en materias afines. Revista Iberoamericana de Educación, 40, 5-25.

Muñoz, Y. (2001). El mercadeo social en Colombia. Medellín: Fondo Editorial Universidad EAFIT.

Ocampo, A. (2011). Inclusión de estudiantes en situación de discapacidad a la educación superior. Desafíos y oportunidades. Revista Latinoamericana de Educación Inclusiva, 6(2), 227-239. 
Porcu, L., Del Barrio-Garcia, S., y Kitchen, P. (2012). How Integrated Marketing Communications (IMC) works? A theoretical review and an analysis of its main drivers and effects/¿Cómo funciona la Comunicación Integrada de Marketing (CIM)? Una revisión teórica y un análisis de sus antecedentes y efectos. Comunicación y sociedad, 25(1), 313- 348.

Rubio, F. (1999). Estrategias de imagen y comunicación en la construcción social de la discapacidad. Revista de Investigación y Marketing, 64, 6-9.

Salinas-Alarcón, M., y Sànchez-Busqués, S. (2014). Actitudes de estudiantes sin discapacidad hacia la inclusión de estudiantes con discapacidad en la educación superior. Barcelona: Universidad Autónoma de Barcelona.

Sánchez, A., Díaz, C., Sanhueza, S., y Friz, M. (2008). Percepciones y actitudes de los estudiantes de pedagogía hacia la inclusión educativa. Estudios pedagógicos (Valdivia), 34(2), 169-178.

Santos Vijande, M. L. (2008). Reseña de" Estrategias y técnicas de comunicación. Una visión integrada en el marketing, de Inma Rodríguez Ardura. Cuadernos de Economía y Dirección de la Empresa, (35), 141-160.

Suriá, R. (2012). Discapacidad e integración educativa: ¿qué opina el profesorado sobre la inclusión de estudiantes con discapacidad en sus clases?. Revista Española de Orientación y Psicopedagogía, 23(3), 96-109.

Suriá, R., Bueno, A., y Rosser, A. (2011). Prejuicios entre los estudiantes hacia las personas con discapacidad: reflexiones a partir del caso de la Universidad de Alicante. Alternativas, 18, 75-90.

Suriá, S., y Villegas E. (2016). Análisis comparativo del empoderamiento entre universitarios con y sin discapacidad. En D. Carbonero, E. Raya, N. Caparros, y C. Gimeno, (Coords) Respuestas transdisciplinares en una sociedad global (pp. 90-100). Logroño: Universidad de la Rioja.

Swain, W. (2004). Perceptions of IMC after a decade of development: Who's at the wheel, and how can we measure success?. Journal of Advertising Research, 44(1), 46-65.

UNESCO. (2009). Directrices sobre políticas de inclusión en la educación. desde http://unesdoc.unesco.org/images/0017/001778/177849s.pdf

Yarza, A., Rojas, H., y López, J. (2013). Discapacidad y Educación Superior: las contiendas por la participación y la ciudadanía en las reformas neoliberales de la Universidad y la educación superior en Colombia. Discapacidad y educación superior, 8(1), 35-39. 